\title{
A inovação nas redes sociais digitais: discurso e prática em empresas de $e$-service
}

\section{Innovation in digital social networks: speech and practice in e-service companies}

\author{
Luana Kava ${ }^{1}$, Rodrigo Eduardo Botelho-Francisco ${ }^{1}$ \\ ${ }^{1}$ Universidade Federal do Paraná, Curitiba, PR, Brasil
}

Autor para correspondência/Mail to: Luana Kava, contato@luanakava.com

Financiamento/Funding: Fundação Araucária e Governo do Estado do Paraná

Copyright (C) 2018 Kava \& Botelho-Francisco. Todo o conteúdo da Revista (incluindo-se instruções, política editorial e modelos) está sob uma licença Creative Commons Atribuição-NãoComercial-Compartilhalgual 3.0 Não Adaptada. Ao serem publicados por esta Revista, os artigos são de livre uso em ambientes educacionais, de pesquisa e não comerciais, com atribuição de autoria obrigatória. Mais informações em http://revistas.ufpr.br/atoz/about/submissions\#copyrightNotice.

\begin{abstract}
Resumo
Este trabalho aborda aspectos de inovação nas redes e mídias sociais nas empresas de e-service (que oferecem serviços de forma online). Neste sentido, visa identificar como acontecem os processos de inovação e como a empresa utiliza o fluxo de informação que surge nas mídias sociais para aprimorar seus produtos, serviços e processos. Especificamente, pretende-se: (a) identificar os fatores de identidade e de engajamento das marcas de $e$-service nas redes sociais digitais; (b) analisar o discurso e a prática de inovação das empresas de $e$-service nas redes sociais; (c) verificar a visão de inovação das empresas de $e$-service nas mídias sociais e; e (d) mapear o fluxo de informação nas empresas de $e$-service. Para alcançar tais objetivos, emprega a Netnografia como perspectiva metodológica para análise das redes sociais digitais. Para coleta e análise de dados utilizam-se análise de conteúdo, entrevista e análise documental. Como resultado, busca-se compreender como as informações advindas das mídias sociais podem possibilitar inovações e se as empresas de e-service possuem a estrutura necessária para utilizar essas informações e desenvolver novos serviços e processos.
\end{abstract}

Palavras-chave: Redes Sociais Digitais; Netnografia; Inovação; Mídias Sociais.

\begin{abstract}
This paper discusses aspects of innovation in networks and social media in e-service (electronic service companies). Hence, it aims to identify how innovation processes happen and how the company uses the information flow that appears in social media to improve its products, services, and processes. Specifically, it is intended to: (a) identify the identity and engagement factors of e-service brands in digital social networks; (b) analyze the discourse and innovation practice of e-service companies in social networks; (c) verify the innovation view of e-service companies in social media; and (d) map the flow of information in e-service companies. To achieve these objectives, it uses netnography as a methodological perspective for the analysis of digital social networks. For data collection and analysis, content analysis, interviewing, and document analysis are used. As a result, it seeks to understand how information coming from social media can enable innovations and whether e-service companies have the framework to use that information and develop new services and processes.
\end{abstract}

Keywords: Digital Social networks; Netnography; Innovation; Social Media.

\section{INTRODUÇÃO}

A inserção de um indivíduo na sociedade se dá por meio das relações que ele estabelece durante sua vida. Como parte da própria natureza humana estão as conexões com outras pessoas (Tomaél, Alcará \& Di Chiara, 2005). Neste sentido, pode-se inferir que estamos essencialmente conectados com outros indivíduos. As redes sociais, que são formadas por atores (tipicamente indivíduos, grupos e empresas) que partilham de valores e objetivos comuns (Mascia, Magnusson \& Bjork, 2015), assim como as redes de comunicação, envolvem a linguagem simbólica, os limites culturais e as relações de poder (Capra, 2002).

Comumente as redes sociais são confundidas com mídias sociais. Estas, apesar de estarem no universo digital, são meios pelos quais uma rede social pode se comunicar (Ciribeli \& Paiva, 2011). Assim, as conexões que são desenvolvidas por meio das mídias sociais são chamadas de redes sociais digitais. Estas são constituídas das representações dos atores sociais e de suas conexões (Recuero, 2009).

As redes sociais possuem historicamente um importante papel nas inovações, na criatividade e na geração de novas ideias (Mascia, Magnusson \& Bjork, 2015). Em contrapartida, as mídias sociais possibilitam a expansão rápida das redes sociais, de forma que os indivíduos podem ser expostos a conteúdos antes não acessíveis (Richey, Ravishankar \& Coupland, 2016).

A criação de uma rede social pode ser feita forma rápida. Esta possibilidade também é valida para as empresas, pois possibilita a busca rápida por sugestões externas, ideias, opiniões e outros atores. Com esta possibilidade de conexão rápida e da geração de novas ideias, as plataformas de mídias sociais podem prover recursos que possibilitam inovação, até mesmo em pequenas e médias empresas (Richey, Ravishankar \& Coupland, 2016; Harris, Rae \& Misner, 2012). 
As redes sociais digitais não proporcionam necessariamente um ambiente equitativo para inovações. Isso acontece porque o processo de inovação nas mídias sociais irá depender das redes e estilos de comportamentos estabelecidos (Richey, Ravishankar \& Coupland, 2016). Quanto maior o número de contatos o indivíduo ou a organização mantém, mais propenso ele é para gerar novas ideias, ao descobrir, combinar e expandir as novas informações (Mascia, Magnusson \& Bjork, 2015; Richey, Ravishankar \& Coupland, 2016).

O termo inovação em mídias sociais, por sua vez, refere-se ao aprimoramento/desenvolvimento de novos produtos e processos que se originaram das redes sociais (Richey, Ravishankar \& Coupland, 2016).

A inovação nas redes sociais foi previamente explorada pela literatura, como, por exemplo, em Richey, Ravishankar and Coupland (2016), que argumentaram que novas formas de conexão nas mídias sociais são cruciais para o processo de inovação que não é facilmente alcançável. Em seu estudo, os autores analisaram de forma qualitativa 31 proprietários de empresas do Reino Unido para identificar as conexões que inspiram as inovações das empresas. Os resultados dos estudos apontaram que a falta de informações disponíveis cria uma certa incerteza sobre o processo de inovação (Richey, Ravishankar \& Coupland, 2016). Vale ressaltar que o objetivo dos autores era de encontrar os pontos iniciais no processo de inovação aberta, por meio de novas conexões nas redes sociais.

Hitchen, Nylund, Ferras and Mussons (2017), também ao abordar este tema, buscaram explorar o uso das redes sociais para o desenvolvimento das inovações abertas e explicar como essas práticas acontecem nas pequenas e médias empresas. Os achados indicam que o uso das tecnologias, como as redes sociais, podem ser transformadas em oportunidades, desafios e estratégias para inovações abertas. Ressalta-se que os autores estudaram no âmbito das inovações abertas e não diretamente na inovação das redes sociais, como o proposto nesta pesquisa.

Já para Rust and Kannan (2003), há empresas que possuem serviços não tão tradicionais, ou seja, são automatizados por meio de um canal virtual e seu objetivo é de direcionar sua atenção aos consumidores, em conhecer suas necessidades e gerar inovações. Este tipo de empresa, que se denomina e-service, é composta por prestação de serviços por meio eletrônico, como por exemplo a venda de passagens aéreas, conferência de entregas, reservas de hotéis, entregas, leilões, softwares, entre outros (Branston \& Stafford, 2010; Rust \& Kannan, 2003). Como a essência das e-services se dá por meio do desenvolvimento tecnológico online e pela busca de desenvolver inovações, acredita-se, nesta pesquisa, que a inovação nas redes sociais destas empresas pode apresentar um resultado mais efetivo em relação ao que a empresa esperava ao inovar.

Com base no levantamento dos estudos relacionados ao tema de inovação nas redes sociais foram identificadas duas lacunas teóricas na literatura. A primeira diz respeito a identificar o processo de inovação em redes sociais em empresas de e-service e a segunda refere-se a compreender os fluxos de informação das organizações para desenvolver inovações com base nas informações advindas das mídias sociais.

Dadas as lacunas teóricas destacadas acima, propõe-se a seguinte questão de pesquisa: Como os processos de inovação em empresas de e-service acontece por meio das redes sociais digitais?

Para responder essa pergunta pretende-se, como objetivo geral desta pesquisa, identificar como os processos de inovação em empresas de e-service acontece nas redes sociais digitais. Especificamente, pretende-se: (a) identificar os fatores de identidade e de engajamento das marcas de e-service nas redes sociais digitais; (b) analisar o discurso e a prática de inovação das empresas de e-service nas redes sociais; (c) verificar a visão de inovação das empresas de e-service nas mídias sociais; e (d) Mapear o fluxo de informação nas empresas de e-service.

O trabalho justifica-se ao mitigar responder às lacunas teóricas identificadas, contribuindo para a literatura especializada ao desvendar os fatores que compõem o processo de inovação nas redes sociais, bem como contribuindo, também, para a literatura de Gestão da Informação, ao mapear os fluxos de informações das empresas em relação às inovações nas redes sociais digitais.

\section{REVISÃO DE LITERATURA}

As redes sociais são compostas por um conjunto de participantes autônomos (também chamados de atores), que unem ideias, recursos em torno de valores e interesses compartilhados (Marteleto, 2001). Pode ser considerada uma das estratégias da sociedade para o compartilhamento de informação e conhecimento mediante as relações entre os atores que as integram (Tomaél, Alcará \& Di Chiara, 2005).

Em uma rede social, cada indivíduo tem sua função e identidade cultural, assim como as relações com os demais membros da rede se apresentam de forma coesa (Tomaél, Alcará \& Di Chiara, 2005). Segundo Capra (2002), as funções e processos sociais na era da informação (na qual vivemos), se organizam cada vez mais em torno de redes.

Von Krogh, Ichijo e Nonaka (2001), salientam que as conversas nas organizações geralmente representam dois objetivos básicos: confirmar o conteúdo do conhecimento ou criar novos conhecimentos. O intercâmbio das ideias, opiniões e crenças propiciado pelas conversas possibilita a criação do conhecimento pelo compartilhamento do conhecimento tácito dentro da comunidade da rede (Krogh, Ichijo \& Nonaka, 2001). 
Há diversos meios para acesso às redes sociais. Podem ocorrer por meio do contato pessoal, do telefone ou, como destaca-se atualmente, via Internet (Ciribeli \& Paiva, 2011). O sucesso do uso deste meio se dá pela liberdade de expressão, a realidade e a confiabilidade dos conteúdos que são ali compartilhados, salvo os cuidados com a segurança pessoal (Ciribeli \& Paiva, 2011).

As redes sociais também devem ser diferenciadas dos meios digitais que as suportam. As mídias sociais são um dos meios digitais pelos quais as comunidades se conectam. Nas mídias sociais os indivíduos falam sobre assuntos pessoais, mas também existe também uma legião de consumidores trocando opiniões sobre produtos e serviços (Ciribeli \& Paiva, 2011). A maioria destas informações são compartilhadas de forma voluntária, o que gera confiabilidade aos demais amigos da redes. Além das informações trocadas pelos amigos, há àquelas que são fornecidas às empresas e dizem respeito à experiência pessoal do usuário (Ciribeli \& Paiva, 2011; Richey, Ravishankar \& Coupland, 2016).

Tsimonis and Dimitriadis (2013) salientam que as empresas buscam se relacionar via redes sociais visando benefícios e resultados efetivos. Estes envolvem principalmente aprimorar o relacionamento com o consumidor, engajamento, implementação das ações de marketing e o poder da marca (Tsimonis \& Dimitriadis, 2013).

É de compreensão das empresas que o uso das mídias sociais pode auxiliar o fortalecimento da marca e na conquista de mais clientes (Ciribeli \& Paiva). Porém, a forma pela qual a empresa gera as informações e desenvolve inovações ainda é um desafio (Richey, Ravishankar \& Coupland, 2016).

Em uma época de competição intensa, complexidade tecnológica e instabilidade institucional, as empresas estão propensas a buscar vantagem competitiva por meio de inovações (Mascia, Magnusson \& Bjork, 2015). Consequentemente, a habilidade de utilizar as informações provenientes das redes sociais se torna essencial para as empresas como fonte potencial de inovação (Mascia, Magnusson \& Bjork, 2015).

Barbosa e Feldmann (2014), apresentam um quadro resumo com as características das empresas inovadoras, tais como: processos de aprendizado, mudança com iniciativas explícitas e continuadas, comprometimento do empreendedor cultura voltada à inovação, foco no consumidor, ambiente propício para a inovação e inovação forte na cadeia de valor. Além destas, Richey, Ravishankar and Coupland (2016) salientam que a inovação pode acontecer nas redes sociais.

O processo de inovação nas redes sociais acontece em três fases: (1) novas conexões, (2) novas informações e (3) inovação (Richey, Ravishankar \& Coupland, 2016). Porém, os autores salientam que há mais aspectos a serem considerados neste processo, pois há muita incerteza por parte das empresas em relação as mídias sociais (Mascia, Magnusson \& Bjork, 2015).

Desta forma, a informação obtida pelas redes sociais possibilita que as empresas gerem inovações. Por outro lado, a empresa para gerar inovações precisa usufruir das informações adquiridas.

\section{PROCEDIMENTOS METODOLÓGICOS}

Conforme salientado nas seções introdutórias deste trabalho, a comunicação por meio de redes sociais e o entendimento das inovação nas mídias sociais são alvo da proposta de pesquisa. Neste contexto surge a perspectiva metodológica da Netnografia, como uma forma especializada de etnografia, de caráter investigativo e de observação da realidade. Trata-se de um método que utiliza de comunicações mediadas por computador como fonte de dados para auxiliar a compreensão e a representação etnográfica de um fenômeno cultural na Internet (Kozinets, 2014).

Para Kozinets (2014, p.66), "a pesquisa em comunidades online tenderia a ter um foco primordialmente netnográfico". Segundo Botelho-Francisco (2018), o método netnográfico colabora para o entendimento dos processos sociais em comunidades online, bem como o compartilhamento de saberes, de experiências e de identidades.

As comunidades on-line passam a ser uma fonte importante de informações sobre as relações sociais dos consumidores entre si e também de consumidor com as marcas (Kozinets, 2014). As empresas de e-service nascem e se desenvolvem essencialmente de forma on-line (Sousa \& Voss, 2006). Com isto, acredita-se que podem criar comunidades online em torno delas. Devido à esta característica nesta pesquisa é proposta o estudo com base as empresas de e-service.

O desenvolvimento de uma pesquisa netnográfica segue quatro etapas específicas, são elas: (a) Entrée cultural; (b) coleta e análise de dados; (c) ética de pesquisa; (d) validação dos resultados obtidos junto à comunidade pesquisada (Kozinets, 2014). Na sequencia será explicitado cada uma das etapas e quais as aplicações para esta pesquisa.

Entrée cultural. Este procedimento é a primeira fase na netnografia e diz respeito à observação não participante e auxilia o pesquisador a definir a comunidade mais adequada para a investigação do problema, ou seja, a preparação para o trabalho de campo (Kozinets, 2014). Definir a comunidade on-line que os sujeitos participam 
auxilia a obtenção da identidade cultural dos integrantes da comunidade (Kozinets, 2014). Nesta etapa, ao definir a comunidade, será definida a amostra de pesquisa. Kozinets (2014) salienta que para a seleção da amostra devem ser considerado que a comunidade seja relevante, interativas substancial, heterogêneas e ricas em dados. Nesta etapa desta pesquisa será definida a amostra de comunidades on-line relacionadas a empresas de $e$-service.

Coleta e análise de dados. Posteriormente a escolha das comunidades on-line, deve ser considerado dois aspectos. Primeiramente, se os dados podem ser coletados diretamente dos discursos entre os membros dessas comunidades. E também, se os dados a serem obtidos se referem à observação sobre a comunidade, seus membros, interações e significados (Kozinets, 2014). Um única comunidade pode gerar uma série de dados a serem classificados. Para classificá-los, Kozinets (2014), sugere que as mensagens sejam classificadas inicialmente como "referente ao tópico" e "fora do tópico". Nesta etapa também será considerada a confiabilidade das interpretações. Como a netnografia é baseada na análise de discursos textuais mediados por meios digitais, para serem confiáveis a análise deve refletir as limitações do ambiente on-line e da técnica (Kozinets, 2014). Na coleta de dados três tipos de capturar são importantes: dados arquivais (cópia direta de conteúdo mediado por computador), dados extraídos (levantados por meio de entrevistas por meio eletrônico) e dados de notas de campo (Kozinets, 2014). Nesta etapa será realizada a coleta e a análise de dados seguindo os padrões sugeridos de uma pesquisa netnográfica.

Ética de pesquisa. A pesquisa netnográfica pressupõe que o pesquisador busque garantir a idoneidade. Levando em consideração a confidencialidade, privacidade e retorno para comunidade (Kozinets, 2014). Kozinets (2014) cita quatro passos importantes para abordar as questões éticas da pesquisa: pedir as permissões apropriadas, obter o consentimento quando necessário, atribuir crédito ao citar membros. Seguindo os padrões de uma netnografia, será realizada a conferência dos padrões éticos da pesquisa.

Validação dos resultados obtidos junto à comunidade pesquisada. Kozinets (2014) aponta dez critérios para validar a pesquisa netnográfica: coerência, rigor, conhecimento, ancoramento, inovação, ressonância, verossimilhança, reflexividade, práxis e miscigenação. Ao realizar a checagem dos dados com os próprios membros do grupo a pesquisa se torna legitima e ganha credibilidade (Kozinets, 2014). Ao validar a pesquisa as conclusões podem ser confirmadas além das observadas em campo. Nesta pesquisa a validação será dada com os membros da comunidade e também na verificação perante as empresas de e-service sobre o uso das informações advindas das redes sociais para o desenvolvimento de inovações.

Com o objetivo de demonstrar as etapas e de distribuir o tempo disponível para a execução do projeto de tese de doutorado aqui proposto, foi elaborado um cronograma (Figura 1). Este cronograma apresenta as etapas a serem realizadas desde o ingresso ao programa de pós-graduação até a defesa da tese.

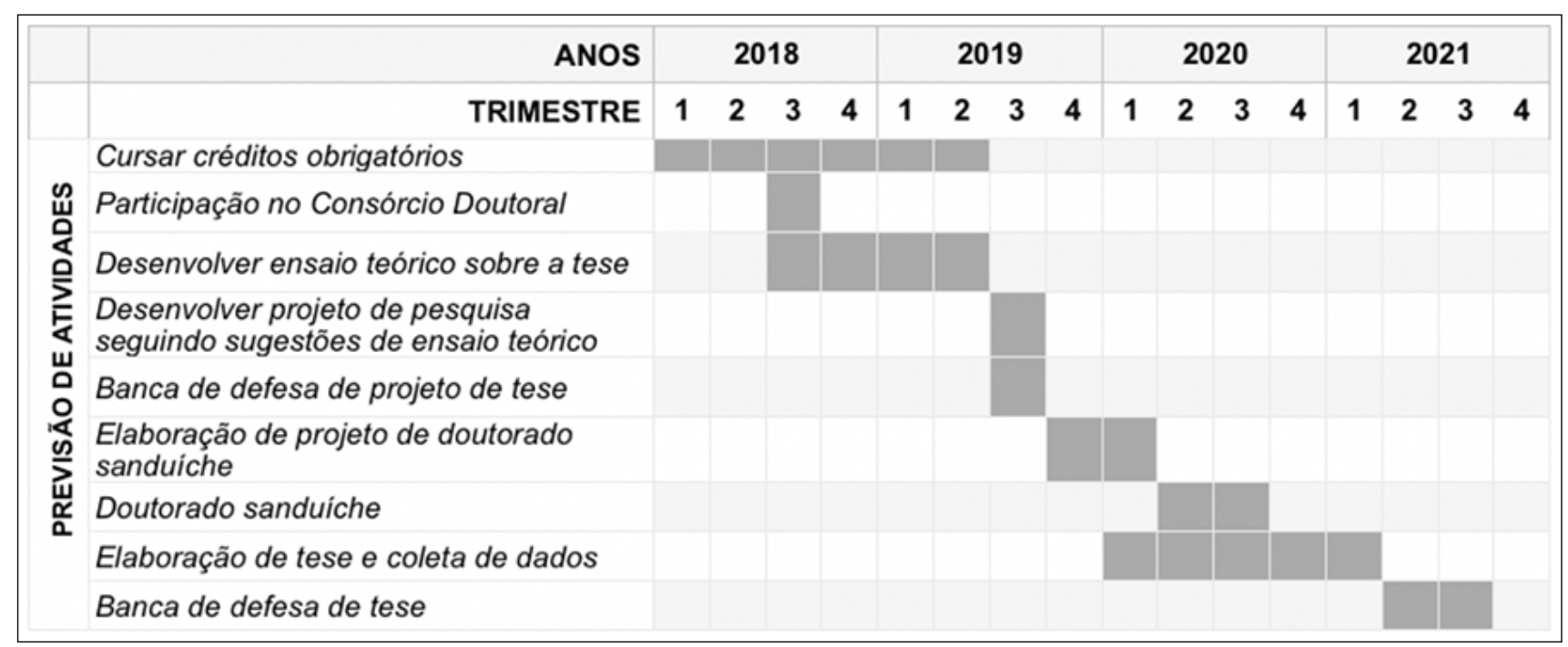

Figura 1. Cronograma de pesquisa

Fonte: Elaborado pelos autores (2018)

Cabe salientar que o cronograma acima apresenta apenas uma estimativa, podendo ser modificado a qualquer momento.

\section{CONSIDERAÇÕES PARCIAIS}

Esta pesquisa netnográfica apresenta como objetivo classificar de que forma acontece os processos de inovação nas empresas de e-service por meio das redes sociais digitais.

Como resultado, busca-se compreender como as informações que surgem nas redes sociais podem possibilitar as inovações nas empresas de e-service. Além disto, ao analisar o fluxo de informações em empresas de e-service, 
pretende-se verificar se as empresas possuem a estrutura necessária para utilizar as informações advindas das redes sociais para desenvolver novos serviços e processos.

Ressalta-se que esta pesquisa está em fase inicial, devido ao recente ingresso da pesquisadora no curso de doutorado, sendo assim se faz necessário aprofundamento e revisão da literatura existente sobre os temas da pesquisa. Em vista disto, as contribuições resultantes à participação deste consórcio doutoral devem contribuir para o desenvolvimento e aperfeiçoamento dessa tese.

\section{AGRADECIMENTOS}
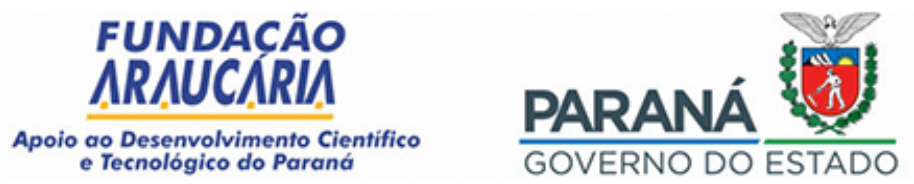

\author{
À Fundação Araucária e ao Governo do \\ Estado pelo apoio financeiro para a rea- \\ lização do evento.
}

\section{REFERÊNCIAS}

Barbosa, A. P. F. P. L., \& Feldmann, P. R. (2014). Características das empresas inovadoras. In Anais do XVII SEMEAD - Seminários em Administração. Recuperado de https://semead.com.br/19/edicoes-anteriores/

Botelho-Francisco, R. E. (2018). Netnografias da comunicação em rede: por uma antropologia do comportamento digital. In A. de C. Azevedo Jr, C. Teixeira Filho; H. W. de C., \& L. J. Cresto. (Org.). Reflexões sobre mídia e consumo. Curitiba: Syntagma Editores, pp. 137-149.

Branston, G., \& Stafford, R. (2010). The media student's book. Reino Unido: Routledge.

Capra, F. (2002). As conexões ocultas. Rio de Janeiro: Cultrix.

Ciribeli, J. P., \& Paiva, V. H. P. (2011). Redes e mídias sociais na internet: realidades e perspectivas de um mundo conectado. Mediação, 13(12). Recuperado de http://www.fumec.br/revistas/mediacao/article/view/509

Harris, L., Rae, A., \& Misner, I. (2012). Punching above their weight: the changing role of networking in SMEs. Journal of Small Business and Enterprise Development, 19(2), 335-351.

Hitchen, E. L., Nylund, P. A., Ferràs, X., \& Mussons, S. (2017). Social media: open innovation in SMEs finds new support. Journal of Business Strategy, 38(3), 21-29. Recuperado de https://doi.org/10.1108/JBS-022016-0015

Kozinets, R. V. (2014). Netnografia: realizando pesquisa etnográfica online. Porto Alegre: Penso.

Marteleto, R. M. (2001). Análise de redes sociais: aplicação nos estudos de transferência da informação. Ciência da informação, 30(1), 71-81. Recuperado de http://www.scielo.br/pdf/ci/v30n1/a09v30n1.pdf

Mascia, D., Magnusson, M., \& Bjork, J. (2015). The role of social networks in organizing ideation, creativity and innovation: an introduction. Creativity and Innovation Management, 24(1), 102-108. Recuperado de https://onlinelibrary.wiley.com/doi/abs/10.1111/caim.12111

Recuero, R. (2009). Redes sociais na internet, difusão de informação e jornalismo: elementos para discussão. In D. de A., \& Soster, F. Firmino. (Org.). Metamorfoses jornalísticas 2: a reconfiguração da forma. Santa Cruz do Sul: UNISC.

Richey, M., Ravishankar, M. N., \& Coupland, C. (2016). Exploring situationally inappropriate social media posts. Information Technology $\&$ People, 29(3), 597-617. Recuperado de https://doi.org/10.1108/ITP-032015-0045

Rust, R. T., \& Kannan, P. K. (2003). e-service: a new paradigm for business in the electronic environment. Communications of the ACM, 46(6), 36-42.

Sousa, R., \& Voss, C. A. (2006). Service quality in multichannel services employing virtual channels. Journal of Service Research, 8(4), 356-371. Recuperado de https://doi.org/10.1177/1094670506286324

Tomaél, M. I., Alcará, A. R., \& Di Chiara, I. G. (2005). Das redes sociais à inovação. Ciência da informação, 34(2). Recuperado de http://revista.ibict.br/ciinf/article/view/1094

Tsimonis, G., \& Dimitriadis, S. (2014). Brand strategies in social media. Marketing Intelligence 8 Planning, 32(3), 328-344. Recuperado de https://doi.org/10.1108/MIP-04-2013-0056

Von Krogh, G., Ichijo, K., \& Nonaka, I. (2001). Facilitando a Criação de Conhecimento: reiventando a empresa com o poder da inovação contínua. Rio de Janeiro: Campus. 